\title{
Downregulation of ribonucleotide reductase subunits M2 induces apoptosis and G1 arrest of cervical cancer cells
}

\author{
NAN WANG ${ }^{1},{\text { YONG } \mathrm{LI}^{2} \text { and JIANHONG ZHOU }}^{1}$ \\ ${ }^{1}$ Laboratory of Molecular Biology, College of Life Sciences, Jiaying University, Meizhou, Guangdong 514015; \\ ${ }^{2}$ Key Laboratory of Molecular Biophysics of The Ministry of Education, College of Life Science and Technology, \\ Center for Human Genome Research, Huazhong University of Science and Technology, Wuhan, Hubei 430074, P.R. China
}

Received February 21, 2017; Accepted November 29, 2017

DOI: $10.3892 / 01.2018 .7806$

\begin{abstract}
Ribonucleotide reductase subunit M2 (RRM2) is associated with the biological behaviours of cancers, including apoptosis, cell proliferation, invasion, cell cycle and migration. Previous studies have suggested that the expression of RRM2 plays critical roles in tumorigenesis in several cancer types. However, the precise molecular mechanism remains unknown. We previously identified RRM2 as a novel downstream target that is activated by human papillomavirus E7, which activates the extracellular signal-regulated kinase $1 / 2$ signalling pathway, but further studies are warranted to establish RRM2 as a therapeutic target. The results of the present study indicate that RRM2 is associated with cervical cancer cell apoptosis and proliferation. The downregulation of RRM2 significantly increased apoptosis, promoted cell cycle arrest at the G1 phase in vitro and inhibited tumour formation in nude mice transplant models in vivo. These results highlight the potential for inhibition of RRM2 expression as a promising therapeutic target for human cervical cancer treatment.
\end{abstract}

\section{Introduction}

Cervical cancer is one of the most common types of malignant tumour and the fourth leading cause of cancer-associated mortality in women worldwide $(1,2)$. Although a cervical cancer vaccine has been successfully developed, cervical cancer is still associated with high worldwide mortality and morbidity rates. At presentation, the majority of cases involve the advanced stage. Cervical carcinogenesis is a multistep process that involves genetic and epigenetic alterations of protein-coding oncogenes, and suppressor genes. A previous study has revealed that persistent infections with high-risk

Correspondence to: Mrs. Nan Wang, Laboratory of Molecular Biology, College of Life Sciences, Jiaying University, Meizhou, Guangdong 514015, P.R. China

E-mail:wang_nan86@163.com

Key words: RRM2, tumorigenesis, apoptosis, cell cycle arrest, cell proliferation human papillomaviruses (HPVs) are the main causes of almost all cervical cancer cases (3). However, a substantial body of evidence has revealed that HPV infections alone are insufficient to induce malignant transformation, and that other genetic alterations may be involved in tumorigenesis and tumour progression (4). Based on this hypothesis, our previous study indicated that the overexpression of ribonucleotide reductase subunit M2 (RRM2), a downstream target gene of HPVE7, promotes cervical carcinogenesis (5).

Human ribonucleotide reductase (RNR) is the rate-limiting enzyme in the production of 2'-deoxyribonucleoside 5 '-diphosphates, and is required for DNA synthesis and repair (6). RNR consists of three subunits, R1, R2 and p53R2. RRM1 contains enzymatically active sites and binding sites for allosteric effectors. RRM2 and p53R2 are 80\% homologous, and both possess a diiron-tyrosyl radical cofactor that is essential for enzyme activity (7). RRM2 is expressed only during the late G1/early $\mathrm{S}$ phase and is degraded in the late $\mathrm{S}$ phase (8). RRM1 interacts with either RRM2 or p53R2 to become the catalytically active form of eukaryotic RR. Therefore, the catalytic activity of RNR is tightly controlled during the cell cycle according to the level of RRM2. Previous studies have demonstrated that the overexpression of RRM2 correlates with cellular invasiveness, metastasis, tumorigenesis and poor patient outcome (9-11). The characteristics of RRM2 that promote tumour progression are associated with its capability to induce the activities of various oncogenes, including those encoding nuclear factor-kB, Myc proto-oncogene protein (c-Myc), tyrosine-protein kinase transforming protein Fes and ornithine decarboxylase $(12,13)$. RRM2 has been reported to be regulated by cell cycle-associated factors, including transcription factor E2F (14). Inhibition of RRM2 expression induces apoptosis and G1/S-phase cell cycle arrest in ovarian cancer (15). Elevated RRM2 expression has been reported to be associated with poor prognosis of cervical cancer. However, the mechanisms by which RRM2 regulates biological functions remain unknown.

Tumorigenesis is the result of uncontrollable cell proliferation, which may be caused by various carcinogenic factors. Several studies have demonstrated that inhibition of cell apoptosis significantly promotes tumorigenesis $(16,17)$. Therefore, understanding the molecular mechanisms that underlie cell apoptosis and cell cycle progression is critical for cancer 
prevention. Cell cycle progression is an important component of controlling cell proliferation (18). The G1- to S-phase transition is a critical stage for cancer formation (19).

To date, few studies have investigated the functions of RRM2 in cervical cancer $(5,20)$. The results of the present study revealed that downregulation of RRM2 in cervical cancer cells increase apoptosis and G1-phase arrest. In addition, RRM2 inhibits cell proliferation. To the best of our knowledge, this study is the first to indicate that RRM2 has a critical role in regulating apoptosis in cervical cancer cells.

\section{Materials and methods}

Cell culture, patient samples and reagents. The human cervical cancer cell lines HeLa, SiHa, CaSki and C33A were obtained from the Type Culture Collection of the Chinese Academy of Sciences (Shanghai, China) and cultured in Dulbecco's modified Eagle's medium (DMEM) supplemented with $10 \%$ fetal bovine serum (Gibco; Thermo Fisher Scientific, Inc., Waltham, MA, USA) at $37^{\circ} \mathrm{C}$ in a humidified $5 \% \mathrm{CO}_{2}$ atmosphere. Low melting temperature agarose were purchased from BD Biosciences, (Franklin Lakes, NJ, USA). Samples from 60 cases of cervical cancer were collected from the Hubei Tumour Hospital (Wuhan, China). All carcinoma tissues were confirmed as invasive cervical cancer and the molecular subtypes of cervical carcinoma were identified based on the 2009 International Federation of Gynaecology and Obstetrics (FIGO) Classification (21). Paracarcinoma tissues were also collected. The present study was approved by the Institutional Review Board of Jiaying University on human subject research and conducted in accordance with the Declaration of Helsinki. Written informed consent was obtained from each study participant. The mouse monoclonal antibodies against RRM2 were purchased from Epitomics (cat. no. 8029-1; Abcam, Cambridge, UK). GAPDH (cat. no. 97166), cyclin-dependent kinase 4 (CDK4; cat. no. 12790), cyclin D1 (cat. no. 2978), phospho-AKT (S473) (cat. no. 4058), AKT (cat. no. 9272), c-Myc (cat. no. 13987), $\beta$-actin (cat. no. 3700), extracellular signal-regulated kinase (ERK; cat. no. 9122) and phospho-ERK1/2 (Thr202/Tyr204) (cat. no. 9101) antibodies were all purchased from Cell Signalling Technology, Inc. (Danvers, MA, USA). Horseradish peroxidase-conjugated secondary antibodies were from Sigma-Aldrich (Merck KGaA; Darmstadt, Germany). The negative control (NC) small interfering siRNA was chemically synthesised from Guangzhou RioboBio Co., Ltd. (Guangzhou, China).

siRNA transfection. The human cervical cancer cells were seeded at $40-50 \%$ confluency and transfected with siRNA or negative control siRNA using Lipofectamine ${ }^{\circledR} 2000$ in Opti-MEM (both from Invitrogen; Thermo Fisher Scientific, Inc.) following the manufacturer's instructions. siRNA was transfected at a concentration of $100 \mathrm{nM}$, while negative control siRNA was transfected at $30 \mathrm{nM}$.

RNA extraction and reverse transcription-quantitative polymerase chain reaction $(R T-q P C R)$. Total RNA was extracted from cells using the TRIzol reagent (Invitrogen; Thermo Fisher Scientific, Inc.), and cDNA was synthesized using the RevertAid First Strand cDNA Synthesis kit K1622
(Thermo Fisher Scientific, Inc.). RT-qPCR was performed using the FastStart Universal SYBR Green Master kit (Roche Diagnostics, Basel, Switzerland) and analysed using an Applied Biosystems 7900 Real-Time PCR system. The concentrations of RNA were determined using a NanoDrop ND-1000 instrument (Thermo Fisher Scientific, Inc.), and aliquots of the samples were stored at $-80^{\circ} \mathrm{C}$. Primer sequences were as follows: GAPDH forward, 5'-TGAACGGGAAGCT CACTGG-3' and reverse, 5'-TCCACCACCCTGTTGCTGT-3'; RRM2 forward, 5'-TTTAGTGAGCTTAGCACAGCGGGA-3' and reverse, 5'-AAATCTGCGTTGAAGCAGTGAGGC-3'. Data analysis was performed using the $2^{-\Delta \Delta C q}$ relative expression quantity method (22).

Protein extraction and western blot analysis. All cells were washed with PBS and lysed with RIPA lysis buffer (Beyotime Institute of Biotechnology, Haimen, China) supplemented with a protease and phosphatase inhibitor cocktail (cat. no. 78440; Thermo Fisher Scientific, Inc.) on ice for $30 \mathrm{~min}$. Cell lysates were centrifuged for $10 \mathrm{~min}\left(12,000 \mathrm{x} \mathrm{g}, 4^{\circ} \mathrm{C}\right)$, the precipitates were collected and the total protein concentrations were determined using the BCA Protein assay kit (Beyotime Institute of Biotechnology). Proteins (40 $\mu \mathrm{g} /$ well) were separated by $12 \%$ SDS-PAGE and transferred to polyvinylidene fluoride membranes (EMD Millipore, Billerica, MA, USA). The membranes were blocked in 5\% non-fat milk for $2 \mathrm{~h}$ at room temperature, then the membranes were cultured at $4^{\circ} \mathrm{C}$ overnight with the primary antibodies CDK4 (1:1,000), GAPDH, cyclin D1, phospho-AKT (S473), AKT, c-Myc, $\beta$-actin, ERK and phospho-ERK1/2 (Thr202/Tyr204) $(1: 2,000)$, rinsed with Tris-buffered saline with Tween-20 (10 mM Tris-Hcl, $150 \mathrm{mM} \mathrm{NaCl}, 0.1 \%$ Tween-20) for three times. Subsequently, membranes were incubated with rabbit anti-mouse (cat. no. A9044) or goat anti-rabbit (cat. no. A0545) secondary antibody (1:20,000; Sigma-Aldrich; Merck KGaA) for $2 \mathrm{~h}$ at room temperature. The signals were detected with the ECL Western Blotting substrate (Thermo Fisher Scientific, Inc.), and protein band intensity was measured using Quantity One software (version 4.52; Bio-Rad Laboratories, Inc., Hercules, CA, USA). Three independent experiments were performed.

Cell cycle analysis. Cervical cancer cells were cultured for $48 \mathrm{~h}$, digested by trypsinization, washed twice in PBS, and fixed with cold $75 \%$ ethanol for $2 \mathrm{~h}$ at $-2^{\circ} \mathrm{C}$. The cells were washed twice in PBS and resuspended in PBS containing DNase-free RNase A $(20 \mu \mathrm{g} / \mathrm{ml})$ and propidium iodide (PI; $50 \mu \mathrm{g} / \mathrm{ml}$ ) (BD Biosciences) in the dark for $30 \mathrm{~min}$ at room temperature. The PI signal was determined by flow cytometry at an excitation wavelength of $488 \mathrm{~nm}$. The results were analysed using ModFit LT ${ }^{\mathrm{TM}}$ software (version 4.1; Verity Software House, Inc., Topsham, ME, USA).

Apoptosis assay. After $48 \mathrm{~h}$ transfections, an Annexin V-fluorescein isothiocyanate (FITC) kit (Nanjing KeyGen Biotech, Co., Ltd., Nanjing, China) was used to assess apoptosis by flow cytometry. Briefly, cells transfected with the siRNA or siRNA mock were resuspended in $100 \mathrm{ml}$ of binding buffer at a density of $1 \times 10^{6}$ cells $/ \mathrm{ml}$, and incubated with Annexin V-FITC for $15 \mathrm{~min}$ at room temperature away from bright light, then PI was added for $5 \mathrm{~min}$ at room temperature. The cells were 
analysed with the Beckman CXP software 2.1 on an FC-500 flow cytometer (both from Beckman Coulter, Inc., Brea, CA, USA) within $1 \mathrm{~h}$ of the cell collection. Apoptosis determination included early apoptotic cells (Annexin V-positive, PI-negative) and late apoptotic cells Annexin V-positive, PI-positive).

Cell proliferation assay. The cervical cancer cells were seeded in 96-well plates at $5 \times 10^{3}$ cells/well and transfected on the following day. Cell proliferation was determined at 24, 48, 72 and 96 h using the CellTiter 96 AQueousOne Solution Cell Proliferation Assay kit (Promega Corporation, Madison, WI, USA), according to the manufacturer's instructions as we previously described (23). The assays were performed in triplicate and were repeated three times.

Colony formation assay. In order to perform the colony formation assay, $1 \mathrm{ml}$ of $0.6 \%$ agarose $+10 \%$ fetal calf serum-DMEM solution (Gibco; Thermo Fisher Scientific, Inc.) was added to six-well plates. Cells transfected with siRRM2 or siNC for $48 \mathrm{~h}$ were trypsinised and seeded into the six-well plates at a density of $5 \times 10^{2}$ cells/well to form natural colonies. After culturing for 14 days at $37^{\circ} \mathrm{C}$, cells were washed twice with PBS, fixed with $4 \%$ methanol for $20 \mathrm{~min}$ at room temperature and stained with haematoxylin for $20 \mathrm{~min}$ at room temperature. Then, the colonies were statistically analysed. The colony formation efficiency (\%) was calculated as the (number of colonies/number of cells seeded) x 100. The experiment was performed in triplicate and repeated at least three times to ensure data reproducibility.

Immunohistochemical staining. Immunohistochemical staining was performed as described in our previous study (21). Briefly, formaldehyde-fixed paraffin-embedded tissue sections were dewaxed in xylene solution and rehydrated using graded ethanol solutions. Antigen retrieval was performed using citrate buffer at $90^{\circ} \mathrm{C}$ for $30 \mathrm{~min}$. The slides were then incubated with RRM2 antibody (1:200; Epitomics, Burlingame, $\mathrm{CA}, \mathrm{USA}$ ) at $4^{\circ} \mathrm{C}$ overnight, and horseradish peroxidase-conjugated secondary antibody (rabbit anti-mouse $\mathrm{IgG}$ ) was added for $30 \mathrm{~min}$ at $37^{\circ} \mathrm{C}$. The colour reaction was developed with 3,3'-diaminobenzidine tetrahydrochloride $/ 0.03 \% \mathrm{H}_{2} \mathrm{O}_{2}$ at room temperature without light for $10 \mathrm{~min}$, followed by counter staining with haematoxylin for 2-4 $\mathrm{min}$ at room temperature.

In vivo xenografts. Four-week-old female BALB/c nude mice $(\mathrm{n}=10$; mean weight was $18 \mathrm{~g}$ ) were purchased from Shanghai SLAC Laboratory Animal Co., Ltd. (Shanghai, China). The animals were housed in a specific pathogen-free environment, and provided sterile food and water ad libitum. Mice were divided into two groups $(n=5)$, either transfected with siRRM2 or negative control siRNA. A total of $5 \times 10^{6}$ HeLa cells suspended in $200 \mu \mathrm{l}$ PBS were subcutaneously injected into the right flanks of the mice. Tumour xenograft diameters were measured using digital calipers twice/week for 35 days, and the tumour volume was calculated using the following formula: Width $^{2} \mathrm{x}$ length $\mathrm{x} 0.5$. On day 35 , the mice were sacrificed and the tumours were excised, fixed in $10 \%$ formalin at $4^{\circ} \mathrm{C}$ for $24 \mathrm{~h}$, and then washed with PBS twice and stored in $0.1 \%$ sodium azide (http://www.bszh.com; Beijing, China) solution for further paraffin embedded slide analysis. All animal experiments were approved by the JiaYing University Animal Care and Use Committee.

Statistical analysis. All data were obtained from at least three independent experiments and are presented as the mean \pm standard deviation. Student's t-test was used to analyse the differences in the means between the two different groups. Data were analysed using GraphPad Prism software (version 6.0; GraphPad Software, Inc., La Jolla, CA, USA). $\mathrm{P}<0.05$ was considered to indicate a statistically significant difference.

\section{Results}

RRM2 expression is associated with cervical carcinogenesis. RRM2 contributes to the malignant cellular phenotype of multiple human cancer types and its overexpression plays a critical role in tumour invasion $(10,12)$. However, little is known regarding its functions in cervical carcinogenesis. To identify the association between RRM2 expression and cervical cancer carcinogenesis, the expression of RRM2 in 38 pairs of specimens was assessed using RT-qPCR. Each bar represents a single patient. The RT-PCR results revealed that the RNA levels of RRM2 were significantly increased in cervical cancer tissues compared with paracarcinoma tissues (Fig. 1A and B). Furthermore, an immunohistochemical analysis of 5 other pairs of cervical cancer samples showed positive staining for RRM2 in all tumour tissues (Fig. 1C). These results demonstrated that the mRNA and protein levels of RRM2 were higher in tumours compared with in paracarcinoma tissues.

RRM2 knockdown inhibits the proliferation of cervical cancer cells in vitro. Our previous study revealed that overexpression of RRM2 induces angiogenesis in cervical cancer (5), but its involvement in other aspects of cervical cancer development remains unclear. To elucidate the biological function of RRM2 in cervical tumorigenesis, RRM2 expression was transiently attenuated using siRNA in cervical cancer cell lines (HeLa, SiHa and CaSki) and the most effective siRNA was chosen for further experiments. The Cell Titer 96 AQueousOne Solution Cell Proliferation assay showed that downregulation of RRM2 significantly inhibited cell proliferation in all three cell lines compared with the control groups (Fig. 2A-C). Similarly, in an anchorage-dependent monolayer colony formation assay, a significant reduction in the colony number of each cell line transfected with siRRM2 compared with the negative control siRNA group was observed (Fig. 2D).

Downregulation of RRM2 inhibits tumorigenesis in vivo. To further investigate the effects of RRM2 on the tumorigenicity of cervical cancer, in vivo tumour formation assays were performed. HeLa cervical cancer cells, which were transfected with siRRM2 or negative control siRNA, were injected into nude mice. Thirty-five days later, the mice were sacrificed, and the tumours were removed and weighed. A significant decrease in xenograft weight and volume was observed in the siRRM2 group (Fig. 3A-C). To further examine the expression of RRM2 in mice treated with siRRM2 or negative control 

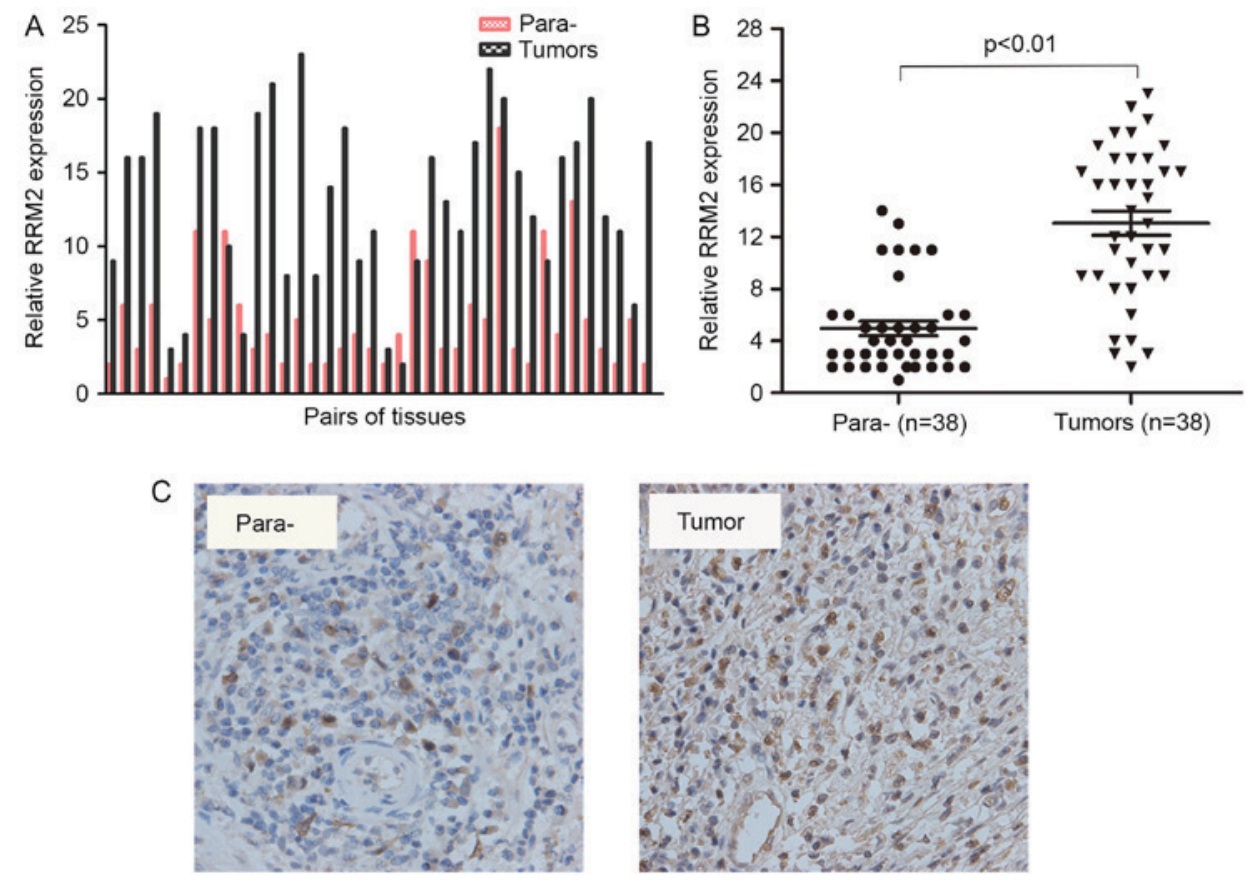

Figure 1. High expression level of RRM2 in cervical cancer patients is associoated with a poor prognosis. (A) Reverse transcription-polymerase chain reaction analysis results of RRM2 in 38 patients with cervical cancer. (B) RRM2 expression was significantly higher in cervical cancer tissues compared with that in the paracarcinoma tissues. (C) Representative images of RRM2 immunohistochemical staining in cervical cancer tissues (right) and paracarcinoma tissues (left). RRM2, ribonucleotide reductase subunit M2.

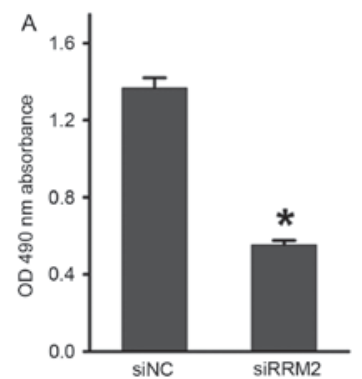

B

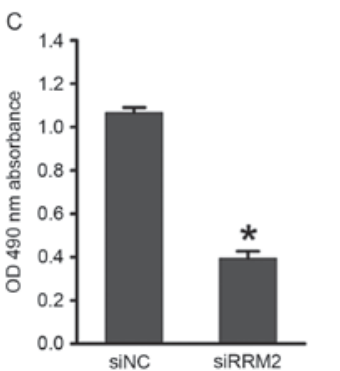

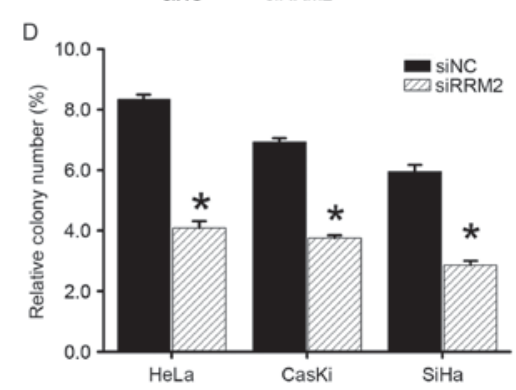

Figure 2. Downregulation of RRM2 inhibits cervical cancer cell proliferation. Analysis of the effect of RRM2 on the proliferation of cells using a CellTiter 96 AQueousOne Solution Cell Proliferation assay in (A) CaSki, (B) HeLa and (C) SiHa cells. (D) The colony formation assay demonstrated the effect of RRM2 on the growth of CaSki, HeLa and SiHa cells. The data represent the mean values of three independent experiments. ${ }^{*} \mathrm{P}<0.05$. RRM2, ribonucleotide reductase subunit M2; si, small interfering RNA; NC, negative control; OD, optical density.

SiRNA, total RNA was extracted from solid tumours and RT-qPCR was performed. The results showed that RRM2 was significantly reduced by siRRM2 (Fig. 3D), which suggested that the downregulation of RRM2 inhibited tumorigenesis in vivo. Therefore, these findings suggest that RRM2 plays an

important role in promoting the malignant growth of cervical cancer cells in vivo.

Downregulation of RRM2 causes cell cycle arrest at the G1 phase. To better understand the growth inhibitory effect of siRRM2 on cervical cancer cell lines, the cell cycle distribution was analysed by flow cytometry. Cervical cancer cell lines were transfected with siRRM2 or negative control siRNA, as shown in Fig. 4A and B. Forty-eight hours after the transient attenuation of RRM2 by siRNA, the percentage of G1-phase cells increased, and the percentage of S-phase cells decreased compared with the percentage of cells from the siRNA control group. This result indicated that the downregulation of RRM2 resulted in cell cycle arrest. To further evaluate the mechanisms underlying cell cycle arrest after the siRRM2 transfection, cell cycle-associated proteins were analysed using western blot analysis. It was demonstrated that active CDK4 and cyclin D1 proteins were markedly downregulated compared with the control group (Fig. 4C). Therefore, it was concluded that the downregulation of RRM2 inhibited the expression of active cyclin D1 and CDK4, leading to cell cycle arrest at the G1 phase in cervical cancer cells.

Downregulation of RRM2 increases apoptosis in cervical cancer cells. Next, the present study focused on the role of RRM2 in regulating cell apoptosis. An Annexin V-FITC apoptosis assay was performed to evaluate the effects of RRM2-mediated apoptosis regulation on cervical cancer tumorigenesis. As shown in Fig. 5A and B, there was an increase in the early and late apoptotic cell populations of HeLa cells after the siRRM2 transfection. The combined percentage of early and late apoptotic siRRM2-treated cells was $\sim 40 \%$, which was significantly increased compared 

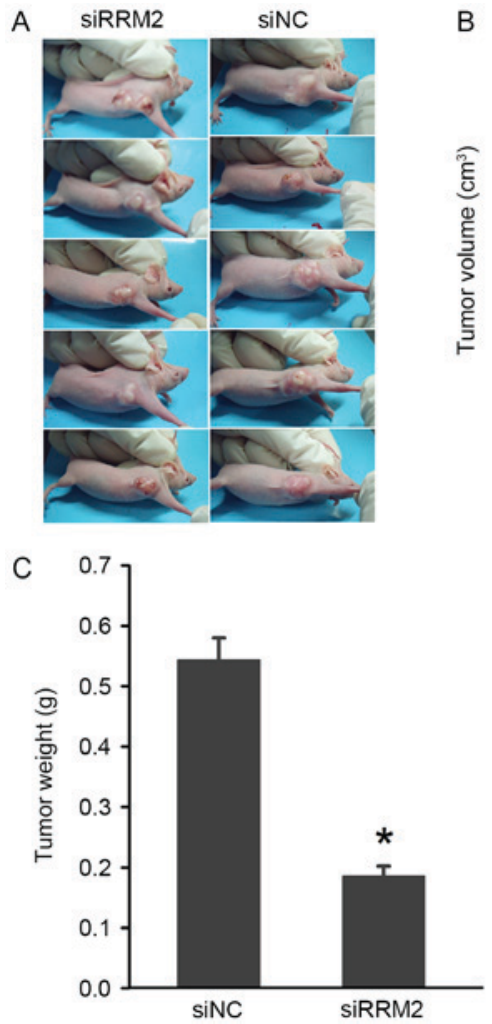
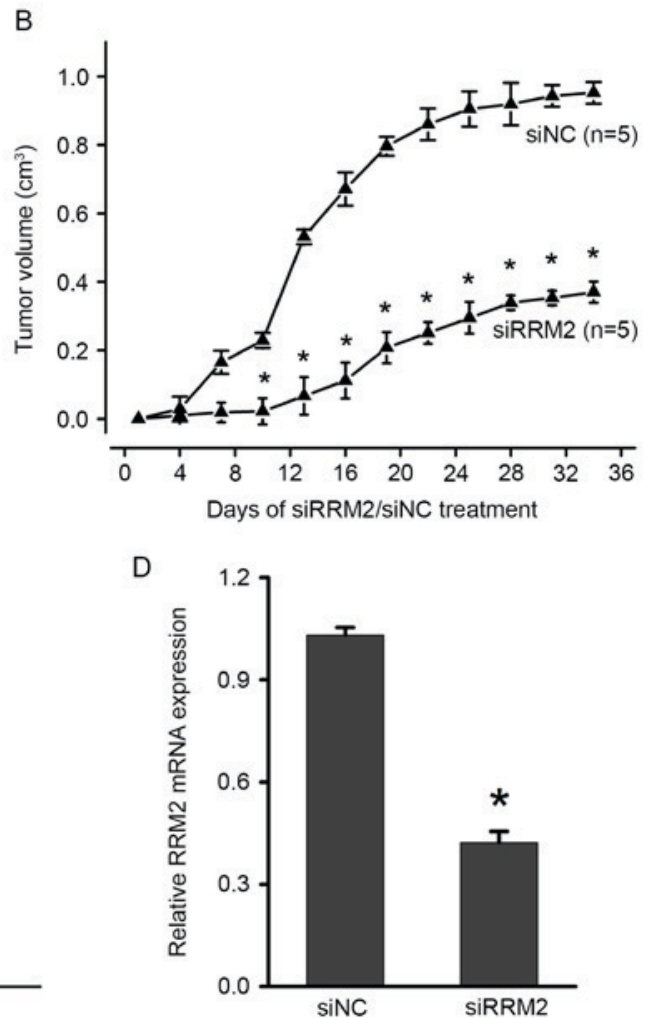

Figure 3. Downregulation of RRM2 suppresses tumorigenesis and progression in nude mice xenograft model. (A) Representative images of the siRRM2 and siNC tumour xenografts. (B) Tumour growth in the siRRM2 group was significantly reduced compared with in the siNC tumour xenograft group after 5 days. (C) Statistical analysis of HeLa xenograft tumour weights at the end of the experiment. (D) RRM2 mRNA expression was analysed by reverse transcription-polymerase chain reaction in different xenograft tissues. "P $<0.05$. RRM2, ribonucleotide reductase subunit M2; si, small interfering RNA; $\mathrm{NC}$, negative control; OD, optical density.

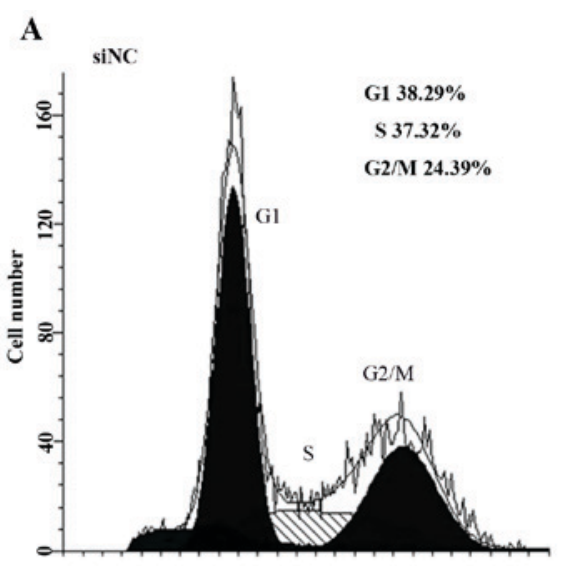

B

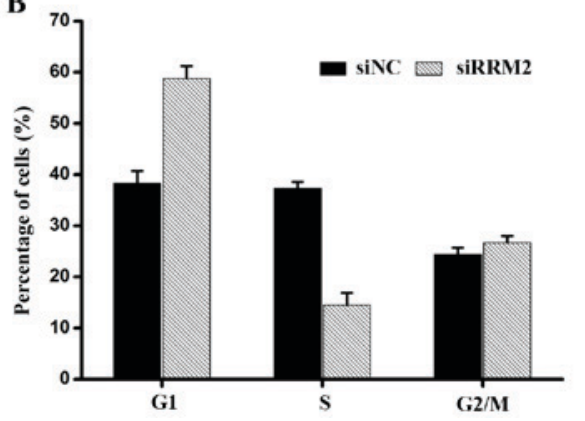

siRRM2

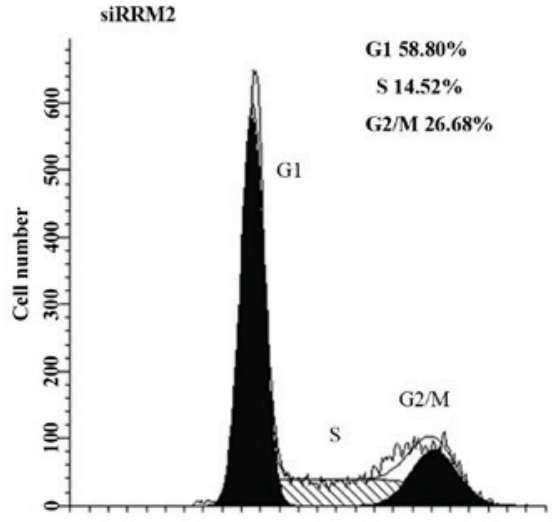

C

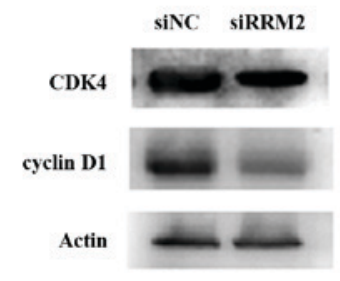

Figure 4. Downregulation of RRM2 in cervical cancer cells induces G1 phase arrest. (A) Cell cycle profiles of HeLa cells transfected with RRM2 siRNA and NC siRNA were determined by flow cytometry and (B) quantified. (C) Representative western blot analysis of G1/S cell cycle regulatory proteins after transfection with RRM2 siRNA and NC siRNA. RRM2, ribonucleotide reductase subunit M2; si, small interfering RNA; NC, negative control; CDK4, cyclin-dependent kinase 4 . 

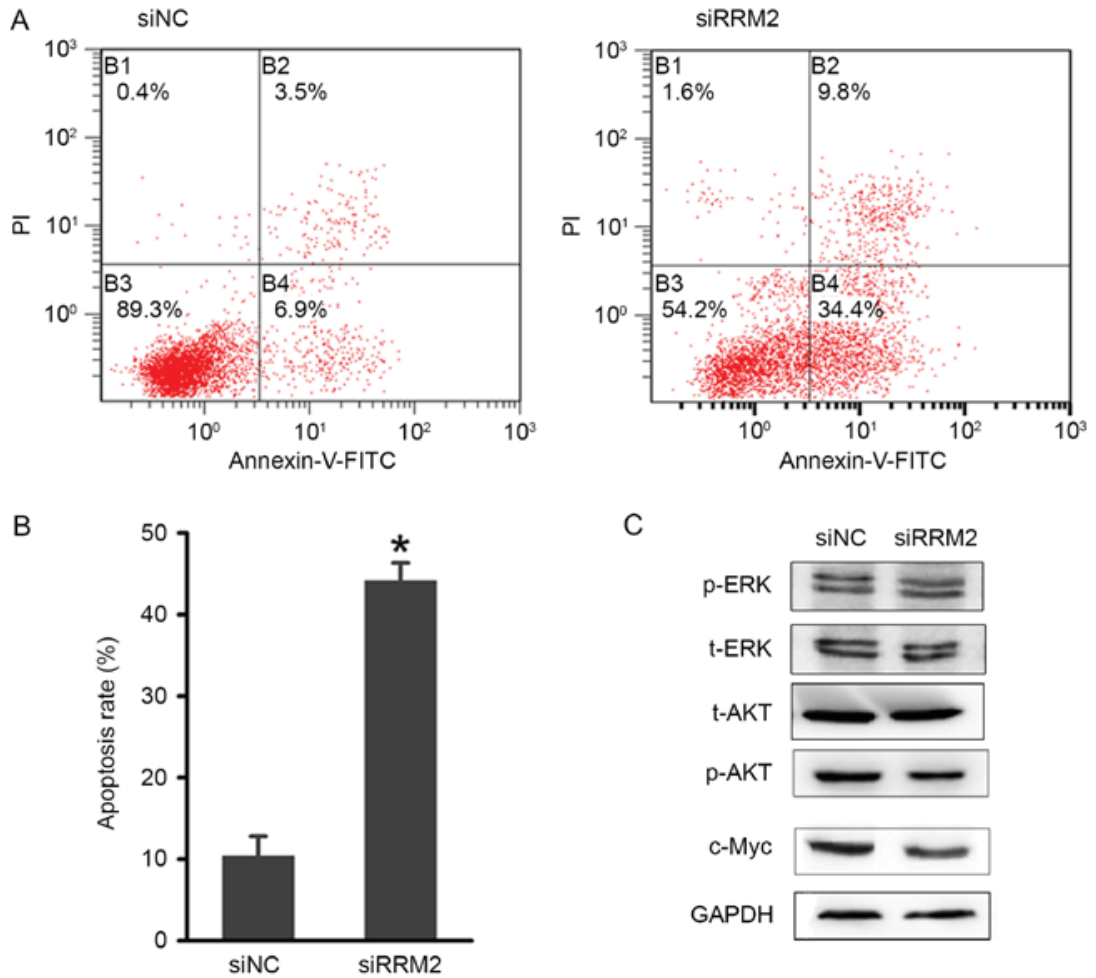

Figure 5. Effect of RRM2 downregulation on cervical cancer cell apoptosis. (A) Flow cytometric analysis of HeLa cell apoptosis with RRM2 siRNA and NC siRNA. (B) Quantitative analysis of the apoptosis rate following transfection. (C) Representative blots demonstrating that RRM2 downregulation reduced p-AKT and c-Myc protein expression. The protein expression level of t-AKT, t-ERK and p-ERK were also analysed by western blotting, but no evident changes were observed. " $\mathrm{P}<0.05$. RRM2, ribonucleotide reductase subunit M2; si, small interfering RNA; NC, negative control; p, phosphorylated; t, total; AKT, AKT serine/threonine kinase; ERK, extracellular signal-regulated kinase; c-Myc, Myc proto-oncogene protein.

with the control group (Fig. 5B). These results suggested that RRM2 suppresses cervical cancer cell growth by inducing G1-phase arrest and apoptosis.

To investigate the potential involvement of signalling mechanisms in the apoptosis and cell cycle arrest that was induced by RRM2 downregulation, western blot analysis of associated proteins was performed. Based on previous studies $(24,25)$, several phosphorylated proteins involved in cell cycle regulation were examined. As shown in Fig. 5C, the phosphorylation of the AKT protein was markedly reduced, while little variation in the phosphorylation of the ERK1/2 protein was observed. The expression of c-Myc was also markedly downregulated. Considering the association between AKT and c-Myc in regulating tumorigenesis, the AKT signalling pathway may be influenced by RRM2.

\section{Discussion}

Accumulating evidence has suggested that RRM2 upregulation is associated with cellular invasiveness (12), metastasis (26), tumorigenesis (10) and poor prognosis (27) in several types of cancer, including colon cancer, pancreatic adenocarcinoma and lung adenocarcinoma. It has been suggested that RRM2 may be a diagnostic and prognostic molecular biomarker, and therapeutic target for cancer in the future. However, the role of RRM2 in the regulation of apoptosis and the cell cycle in cervical cancer has not been elucidated. To the best of our knowledge, the results of the present study presented a previously unknown role for RRM2 in cervical carcinogenesis. The results of this study suggest that RRM2 may be a promising target for the treatment of cervical cancer.

In the present study, it was demonstrated that RRM2 mRNA was significantly upregulated in cervical cancer compared with paracarcinoma tissues. RRM2 knockdown by siRNA significantly inhibited cervical cancer cell proliferation and induced cell apoptosis in vitro. Based on the in vitro results, the role of siRRM2 in tumorigenesis in nude mice was examined. It was demonstrated that downregulation of RRM2 prevented xenograft tumour growth in vivo, suggesting that decreased RRM2 expression inhibited tumorigenesis in cervical cancer. In line with these results, the cell cycle distribution was further analysed to investigate the mechanisms underlying the growth suppressive role of siRRM2. It was revealed that RRM2 knockdown inhibited cell cycle progression at the G1 phase through the AKT signalling pathway in cervical cancer cells, and CDK4 and cyclin D1 protein expression was downregulated. In addition, c-Myc expression was markedly downregulated. Considering the association between AKT and c-Myc in regulating tumorigenesis, further studies are needed to provide further insights into the detailed molecular mechanisms by which RRM2 decreases activation of the AKT signalling pathway.

RRM2 knockdown was shown to reduce cell proliferation and the invasive ability in gastric cancer (28). In addition, silencing of RRM2 expression in pancreatic adenocarcinoma was demonstrated to attenuate cellular invasiveness (12). In the present study, it was revealed that the downregulation of RRM2 significantly induced apoptosis and prevented cell cycle 
progression at the $\mathrm{G} 1$ phase in cervical cancer cells. These results suggest that RRM2 has a significant role in driving tumour cell invasion and metastasis. However, whether the effect of RRM2 in regulating cervical cancer cell apoptosis, and cell cycle arrest is time- and concentration-dependent will need to be elucidated. Assays that assess the effect of RRM2 in metastasis in vivo should be addressed in future studies to fully understand its biological functions in cervical cancer progression.

Several studies have shown that RRM2 is an independent prognostic factor and may predict poor survival for certain cancer types (29-31). However, few studies have elucidated the underlying mechanisms of RRM2 in cervical cancer. To the best of our knowledge, the results of the present study are the first to demonstrate that the downregulation of RRM2 inhibits cervical cancer tumorigenesis and progression. This may aid in improving the understanding of the molecular mechanisms underlying cervical cancer. It is of interest to further investigate whether RRM2 is able to act as a biomarker for predicting the cervical cancer prognosis and patient survival in the future.

\section{Acknowledgements}

The present study was supported by the Chinese National Natural Science Foundation (grant no. 81502257) and by the Initial Scientific Research Fund of Young Teachers in Jiaying University (grant no. 311A0409).

\section{References}

1. Bodily JM, Mehta KP and Laimins LA: Human papillomavirus E7 enhances hypoxia-inducible factor 1-mediated transcription by inhibiting binding of histone deacetylases. Cancer Res 71: 1187-1195, 2011.

2. Jemal A, Bray F, Center MM, Ferlay J, Ward E and Forman D: Global cancer statistics. CA Cancer J Clin 61: 69-90, 2011.

3. Hildesheim A and Wang SS: Host and viral genetics and risk of cervical cancer: A review. Virus Res 89: 229-240, 2002.

4. Mansur CP and Androphy EJ: Cellular transformation by papillomavirus oncoproteins. Biochim Biophys Acta 1155: 323-345, 1993.

5. Wang N, Zhan T, Ke T, Huang X, Ke D, Wang Q and Li H: Increased expression of RRM2 by human papillomavirus E7 oncoprotein promotes angiogenesis in cervical cancer. $\mathrm{Br} \mathrm{J}$ Cancer 110: 1034-44, 2014.

6. Nordlund P and Reichard P: Ribonucleotide reductases. Annu Rev Biochem 75: 681-706, 2006.

7. Tanaka H, Arakawa H, Yamaguchi T, Shiraishi K, Fukuda S, Matsui K, Takei Y and Nakamura Y: A ribonucleotide reductase gene involved in a p53-dependent cell-cycle checkpoint for DNA damage. Nature 404: 42-49, 2000.

8. Engström Y, Eriksson S, Jildevik I, Skog S, Thelander L and Tribukait B: Cell cycle-dependent expression of mammalian ribonucleotide reductase. Differential regulation of the two subunits. J Biol Chem 260: 9114-9116, 1985.

9. Zhou BS, Tsai P, Ker R, Tsai J, Ho R, Yu J, Shih J and Yen Y: Overexpression oftransfected human ribonucleotide reductase M2 subunitin human cancer cells enhances their invasive potential. Clin Exp Metastasis 16: 43-49, 1998.

10. Zhang K, Hu S, Wu J, Chen L, Lu J, Wang X, Liu X, Zhou B and Yen Y: Overexpression of RRM2 decreases thrombspondin-1 and increases VEGF production in human cancer cells in vitro and in vivo: Implication of RRM2 in angiogenesis. Mol Cancer 8: $11-22,2009$.

11. Liu X, Zhang H, Lai L, Wang X, Loera S, Xue L, He H, Zhang K, Hu S, Huang Y, et al: Ribonucleotide reductase small subunit M2 serves as a prognostic biomarker and predicts poor survival of colorectal cancers. Clin Sci (Lond) 124: 567-578, 2013.
12. Duxbury MS and Whang EE: RRM2 induces NF-kappaBdependent MMP-9 activation and enhances cellular invasiveness. Biochem Biophys Res Commun 354: 190-196, 2007.

13. Fan H, Villegas C, Huang A and Wright JA: The mammalian ribonucleotide reductase R2 component cooperates with a variety of oncogenes in mechanisms of cellular transformation. Cancer Res 58: 1650-1653, 1998.

14. Chabes AL, Björklund S and Thelander L: S Phase-specific transcription of the mouse ribonucleotide reductase $\mathrm{R} 2$ gene requires both a proximal repressive E2F-binding site and an upstream promoter activating region. J Biol Chem 279: 10796-10807, 2004.

15. Zhang M, Wang J, Yao R and Wang L: Small interfering RNA (siRNA)-mediated silencing of the M2 subunit of ribonucleotide reductase: A novel therapeutic strategy in ovarian cancer. Int J Gynecol Cancer 23: 659-666, 2013.

16. Hanahan D and Weinberg RA: The hallmarks of cancer. Cell 100: 57-70, 2000.

17. Fulda S and Vucic D: Targeting IAP proteins for therapeutic intervention in cancer. Nat Rev Drug Discov 11: 109-124, 2012.

18. Vermeulen K, Van Bockstaele DR and Berneman ZN: The cell cycle: A review of regulation, deregulation and therapeutic targets in cancer. Cell Prolif 36: 131-149, 2003.

19. Chen HZ, Tsai SY and Leone G: Emerging roles of E2Fs in cancer: An exit from cell cycle control. Nat Rev Cancer 9: 785-797, 2009.

20. Su YF, Wu TF, Ko JL, Tsai HT, Tee YT, Chien MH, Chou CH, Lin WL, Low HY, Chou MY, et al: The expression of ribonucleotide reductase M2 in the carcinogenesis of uterine cervix and its relationship with clinicopathological characteristics and prognosis of cancer patients. PLoS One 9: e 91644, 2014.

21. Pecorelli S: Revised FIGO staging for carcinoma of the vulva, cervix, and endometrium. Int J Gynaecol Obstet 105: 103-104, 2009.

22. Livak KJ and Schmittgen TD: Analysis of relative gene expression data using real-time quantitative PCR and the 2(-Delta Delta C(T)) method. Methods 25: 402-408, 2001.

23. Wang N, Zhou Y, Zheng L and Li H: miR-31 is an independent prognostic factor and functions as an oncomir in cervical cancer via targeting ARID1A. Gynecol Oncol 134: 129-137, 2014.

24. Molina JR and Adjei AA: The Ras/Raf/MAPK pathway. J Thorac Oncol 1: 7-9, 2006.

25. Li XD, Zhang YJ and Han JC: Betulin inhibits lung carcinoma proliferation through activation of AMPK signaling. Tumour Bio 135: 11153-11158, 2014

26. Liu X, Zhou B, Xue L, Yen F, Chu P, Un F and Yen Y: Ribonucleotide reductase subunits M2 and p53R2 are potential biomarkers for metastasis of colon cancer. Clin Colorectal Cancer 6: 374-381, 2007.

27. Souglakos J, Boukovinas I, Taron M, Mendez P, Mavroudis D, Tripaki M, Hatzidaki D, Koutsopoulos A, Stathopoulos E, Georgoulias V and Rosell R: Ribonucleotide reductase subunits M1 and M2 mRNA expression levels and clinical outcome of lung adenocarcinoma patients treated with docetaxel/gemcitabine. Br J Cancer 98: 1710-1715, 2008.

28. Kang W, Tong JH, Chan AW, Zhao J, Wang S, Dong Y, Sin FM, Yeung S, Cheng AS, Yu J and To K: Targeting ribonucleotide reductase M2 subunit by small interfering RNA exerts anti-oncogenic effects in gastric adenocarcinoma. Oncol Rep 31: 2579-2586, 2014

29. Ferrandina G, Mey V, Nannizzi S, Ricciardi S, Petrillo M, Ferlini C, Danesi R, Scambia G and Del Tacca M: Expression of nucleoside transporters, deoxycitidine kinase, ribonucleotide reductase regulatory subunits, and gemcitabine catabolic enzymes in primary ovarian cancer. Cancer Chemother Pharmacol 65: 679-686, 2010.

30. Kretschxmer C, Sterner-Kock A, Siedentopf F, Schoenegg W, Schlag PM and Kemmner W: Identification of early molecular markers for breast cancer. Mol Cancer 10: 15, 2011.

31. Morikawa T, Maeda D, Kume H, Homma Y and Fukayama M: Ribonucleotide reductase M2 subunit is a novel diagnostic marker and a potential therapeutic target in bladder cancer. Histopathology 57: 885-892, 2010.

This work is licensed under a Creative Commons Attribution-NonCommercial-NoDerivatives 4.0 International (CC BY-NC-ND 4.0) License. 